\title{
Last Study Treatment Time
}

National Cancer Institute

\section{Source}

National Cancer Institute. Last Study Treatment Time. NCI Thesaurus. Code C162170.

The time of the subject's final study treatment. 